\title{
Versorgung ohne Schwelle
}

\section{Das Quartier als Milieu der Dienste und des Engagements}

\author{
WOLF RAINER WENDT \\ Prof. Dr. Wolf Rainer Wendt ist Vor- \\ sitzender der Deutschen Gesellschaft \\ für Care und Case Management \\ sowie Honorarprofessor der Eberhard \\ Karls Universität Tübingen. Er ist \\ Mitbegründer und langjähriger Vor- \\ sitzender der Deutschen Gesellschaft \\ für Soziale Arbeit. Seit über drei Jahr- \\ zehnte ist er Mitglied im Beirat der \\ Blätter der Wohlfahrtspflege. \\ E-Mail prof.dr.wendt@googlemail.com
}

\author{
Die Kategorien von Person, Milieu und Sozialraum \\ beschreiben eine komplizierte Konstellation, in der \\ sich professionelle soziale Dienste bewegen und \\ Einrichtungen der Wohlfahrt ihr Angebot bereithalten. \\ Sie müssen sich dazu kommunikativ auf die Spezifik der \\ Lebensführung ihrer Adressaten beziehen. Anzustreben \\ ist eine "Inklusion der Dienste» in die Bürgerkommune \\ mit einem hohen Maß an Selbstregulierung.
}

Der Sozialraum wird als Feld der Betätigung von Sozialdiensten wahrgenommen. Das ist aber nicht die gleiche Perspektive wie die eines lokalen bürgerschaftlichen Engagements und deckt sich nicht mit dem Gesichtskreis, in dem die Menschen im Quartier ihre Verhältnisse erfahren und ihr Leben gestalten.

Steht die Chiffre "Sozialraum« oft nur für einen anderen Zugang der Sozialverwaltung und von Fachagenturen zu Ressourcen vor Ort und für eine neue Steuerung des Einsatzes von Diensten, so rückt mit der Wahrnehmung lokaler Milieus das Lebensfeld der Bewohner mit deren Sicht, ihrer Eigensorge und sozialräumlichen Betätigung in den Fokus einer gestaltenden Arbeit. Voraussetzung ist, dass die Dienste milieukonform werden.

\section{Handeln im Feld oder ankommen im Milieu}

Im Nahraum des Lebens und Wohnens gibt es viele Hilfen für Jung und Alt, für Familien und für Menschen mit Behinderungen. Die Infrastruktur der sozialen Versorgung liegt den potenziellen Nutzern, die jene Hilfen nötig haben, aber nicht wirklich nahe. Die Dienste und Einrichtungen sind formal organisiert und arbeiten professionell. Das scheidet sie vom Leben um sie herum. Die Fachagenturen machen sich mit dem Milieu, in dem es sie gibt, nicht gemein. Dazu müssten sie im Sozial- raum »mittendrin «, verwoben mit dem Alltag in ihm sein und als ihm und seinem Beziehungsgefüge zugehörig erlebt werden. Kann so formelle Versorgung gewissermaßen informell »zur Stelle sein« für die Bewohner eines Quartiers?

Alltägliche Lebensführung differiert und zieht eigene Kreise. Es gibt viele und verschiedene Milieus in dem Sinne, dass es Lebensauffassungen und Lebensweisen sind, die ein soziales Umfeld prägen. Die vom Sinus-Institut (www.sinus-institut.de/loesungen/sinus-milieus.html) in einem Milieu-Raster erfassten Bevölkerungsgruppen unterscheiden sich in ihrer Lebenswelt »von innen heraus «: Sie werden in ihren Befindlichkeiten und Orientierungen, Werten, Lebenszielen und Lebensstilen beschrieben. Der Horizont ihrer Lebenswelt ist ein je anderer. Vom konservativ-etablierten Milieu über die bürgerliche Mitte der Leistungs- und Anpassungsbereiten bis zum prekären Milieu einer "um Teilhabe bemühten Unterschicht « sind zehn Milieus in diesem Modell ausgemacht worden. In ihnen - und zwar individuell und kaum kollektiv ausgeprägt - dürften die Erwartungen an die Gestaltung sozialer Teilhabe und sozialer Versorgung jeweils andere sein.

Diese Varianz betrifft auch die Jugendkulturen, die auf ihr Umfeld unterschiedlich angewiesen sind und es verschieden nutzen (Krisch 2009. 128 ff.). Soziale Arbeit mit Jugendlichen sollte in differenzierender Weise deren Eigenleis- 


\section{"Wir sind das Dorf»}

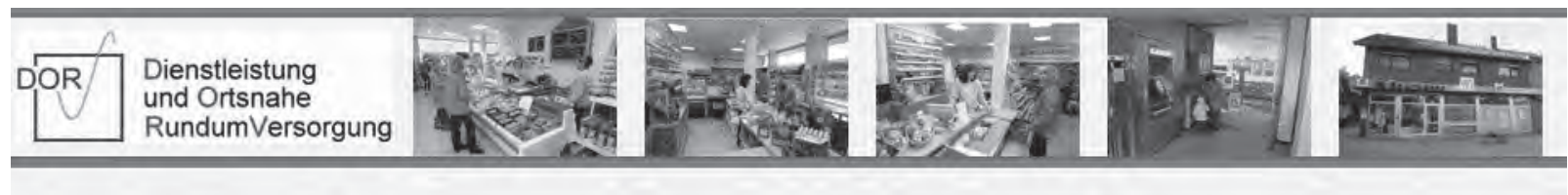

Von dem kleinen niederrheinischen Dorf Barmen ist 2004 die Gründung von DORV-Zentren ausgegangen, wobei das Kürzel für "Dienstleistung und Ortsnahe Rundum Versorgung" steht. Dort, wo es an Infrastruktur mangelt, weil es sich für kommerzielle Anbieter nicht lohnt, sind aus bürgerschaftlicher Initiative (1.) die Nahversorgung mit Lebensmitteln und Gütern des täglichen Bedarfs, (2.) öffentliche und private Dienstleistungen, (3.) eine Servicestation mit medizinischen Versorgungs-, pflegerischen und sozialen Beratungs- und Vermittlungsleistungen sowie ein Veranstaltungsort für (4.) Kommunikation und (5.) kulturelle Aktivitäten zusammengeführt worden ("5-SäulenModell«). Dienste und Gemeindeleben verbinden sich. Charakteristisch für die DORV-Zentren ist, dass die Gestaltung der Versorgung "rundum« von den Bürgern (»Wir sind das Dorf getragen wird - mit Partnern seitens der Kommune und verschiedener Sozialleistungsträger.

Internet http://www.dorv.de

„Im Laden kauft man nicht nur ein. Er ist ein soziales Zentrum, wo man andere trifft, ein bisschen klönt, Neuigkeiten aus dem
Dorf austauscht", sagt Heinz Frey, Lehrer und Architekt aus dem nordrhein-westfälischen Jülich-Barmen. Dass es den Laden im Ort noch gibt, hat Frey sich selbst und einer Handvoll Engagierter zu verdanken. In den goer-Jahren sperrten die beiden Metzger zu, der Lebensmittelladen, der Bäcker. 2001 gab die Sparkasse auf. Die Reaktion der Dörfler war Enttäuschung, dann Wut. Und zuletzt: Einfallsreichtum.

Frey und einige andere nahmen ihr Schicksal in die Hand. Sie gaben Bürgeraktien à 250 Euro aus und bekamen so inklusive Krediten 100.000 Euro zusammen. Damit eröffneten sie vor sechs Jahren einen Laden mit mehreren fest angestellten Verkäuferinnen, mit einem Zeitschriftenregal und Schreibwaren, Fleischtheke, Brottheke, Frischobst und Kühlregal. Dazu eine Paketannahme und eine zwei Wochentage besetzte Arztpraxis. Ältere Dorfbewohner bekommen um die Ecke wieder alles, was sie zum Leben brauchen. Und die Hauptstraße von JülichBarmen lebt wieder.

Quelle: Die Welt vom 10. Oktober 2010 tungen würdigen und an ihnen mit einer »>Pädagogik der Anerkennung` anschließen« (Thomas/Calmbach 2011, 15).

\section{Orientierung im Raum reicht nicht}

Wenn das Milieu ein Horizont ist, in dem Menschen ihr Leben gestalten und damit ihre Probleme haben, können wir ein Milieu nicht einfach mit dem physischen Raum gleichsetzen. In ihm existieren oft verschiedene $\mathrm{Mi}$ lieus nebeneinander. Entsprechend ist die Beziehung von Diensten auf sie eine je andere, weil die Bedarfe, die Interessen und die Zugangsweisen variieren. Der Raumbezug als solcher bringt nicht schon Angemessenheit des dienstlichen Einsatzes mit sich.

Die Ausrichtung von Diensten (der Jugendhilfe) auf den Sozialraum ist als eine »vom Fall zum Feld « unter Beiziehung der fallunabhängig und fallübergreifend im Quartier erschließbaren Ressourcen verstanden worden. Danach organisieren die Verwaltung (das Jugendamt) und die Fachkräfte ihre Arbeit dezentral und in einem Netzwerk lokaler Akteure.

Analog greifen Pflegestützpunkte nach $\mathbb{S} 92$ c SGB IX die im Nahraum vorhandenen Angebote an professionel- len und informellen Hilfen und diversen Versorgungsmöglichkeiten auf. Hier ist $\mathrm{zu}$ differenzieren. Viele Bürger finden von sich aus Zugang zu Rat in ihrem Umfeld und zu Unterstützung in vernetzten Dienstleistungsstrukturen. Andere nicht. Ihnen können Wege gebahnt werden. Milieuspezifisch erweitern und erleichtern die Möglichkeiten virtueller Vernetzung einen Zugang.

Nähe im Austausch, in achtsamer Wahrnehmung von Problemen, in gemeinsamer Beratung und mit umstandslos erreichbarer Aushilfe wird unter Zurückstellung der Expertise erreicht, die ein Dienst mitbringt und die sich zuerst der Prüfung aussetzen muss, wo und wie sie tatsächlich gebraucht wird. Dazu mischt sich der Dienst, bevor er am Einzelfall ansetzt, schon in informelle Begegnungen, bei lokalen Treffen und kommunalen Veranstaltungen ein, zu denen Bürger mit ihren unterschiedlichen Interessen zusammenkommen.

In der Sozialen Arbeit mit Randgruppen ist das längst Praxis. Zum Beispiel pflegt man in der Straßensozialarbeit mit bestimmten Problemgruppen sehr weit in der Identifikation mit der Klientel zu gehen, um von ihr angenommen zu werden. Andere Milieus erfordern andere Praktiken. Sie stoßen auf eine unterschiedliche Politik der Führung des Lebens (»life politics«, Anthony Giddens). Darin können Dienste ihren Platz finden, wenn sie sich kommunikativ auf die Spezifik der Lebensführung ihrer Adressaten beziehen. Anzustreben ist allgemein eine »Inklusion der Dienste" in die Bürgerkommune mit einem hohen Maß an Selbstregulierung (vgl. Holtkamp/Bogumil 2007).

\section{Ein "Wir-Raum» nach eigener Wahl}

Im Fachdiskurs zum Sozialraum der letzten Jahre ist man bald darauf gekommen, ihn nicht mehr nur als Feld der Administration, der Planung, des Zugriffs auf Ressourcen und der Intervention zu begreifen.

Unter dem Anspruch »Den Sozialraum vom Menschen her denken!« (Reutlinger 2006, 23) lässt sich der Blick auf die den Raum ihres Lebens konstituierenden Subjekte richten. Junge Menschen eignen ihn sich an und bewegen sich in ihm als einem erweiterten Zuhause. Cliquen treten im Aktionsgeflecht einer "Szene « auf (siehe des Näheren Krisch 2009). Klaus Dörner spricht im Kontext des Lebens im Alter von einem »dritten Sozialraum«, 
der sich zwischen den Sozialraum des Privaten und den Sozialraum des Öffentlichen schiebt und ein "Wir-Raum" von Nachbarn ist. (Dörner 2007, 2010). Exemplarisch nennt Dörner Beziehungen der Versorgung unter Älteren, die sie in "Wahlverwandtschaft " gestalten, und die Wohnpflegegruppen, die in einer Reihe von Städten die ambulante Pflegeversorgung auf eine neue Basis gestellt haben. Was Professionelle nicht $\mathrm{zu}$ leisten vermögen, leistet man sich in selbstgewählter Gemeinschaft. "Nur Bürger können Bürger integrieren «, hat Dörner als Leitsatz ausgegeben.

An Initiativen der Selbstgestaltung können sich Wohlfahrtsorganisationen mit der Entwicklung quartiersnaher Wohn- und Assistenzangebote anschließen, wie sie etwa das "Netzwerk Soziales neu gestalten $(\mathrm{SONG})$ « in die Wege geleitet hat. Schrittweise wird dabei zu sozialraumorientierter Assistenz in lokaler Kooperation mit engagierten und betroffenen Bürgern übergegangen. Ein Beispiel unter mehreren zu diesem Hilfemix (Zukunft Quartier 2007) bietet die Altenhilfe der Stiftung Liebenau, die ihr Konzept »Lebensräume für Jung und Alt « in einer Verbindung von Selbst- und Nachbarschaftshilfe, mit Gemeinwesenarbeit und einem Servicezentrum realisiert. (Schiele 2011) Analog wird die Chiffre »Lebensraum» für einen Freiraum der Selbstgestaltung gewählt, den Familien oder behinderte Menschen in Partnerschaft mit professionellen Hilfen nutzen können.

\section{In das Umfeld integriert}

Zunächst nicht von der Pflege im Alter her, sondern in der Jugendhilfe hat man den Sozialraum als Feld selbstaktiver Problemlösung entdeckt. An dieses Aktivitätsspektrum lässt sich dienstlich subsidiär anschließen. Das von Wolfgang Hinte vertretene Fachkonzept der Sozialraumorientierung (Hinte/Treeß 2011) fokussiert auf Arrangements, die dazu beitragen, dass Menschen in ihrer Lebenswelt und problematischen Situation zurechtkommen. Fünf Handlungsprinzipien charakterisieren dieses Konzept:

- Ausgang von den Interessen und dem Willen der Menschen

- Vorrang von "aktivierender Arbeit" vor Betreuung
- Nutzung personaler und sozialräumlicher Ressourcen

- Gestaltung der Arbeit bereichs- und zielgruppenübergreifend

- Vernetzung und Integration von Diensten im Einzelfall

Hinte hebt den Willen einer Person von den Wünschen ab, die sie haben mögen, und konkretisiert den Willen in einem wirklichen Interesse und in der Entschiedenheit: »Ich bin entschlossen, mit eigener Aktivität zum Erreichen meines Zieles beizutragen « (Hinte 2011, 225). Eigenes Engagement im persönlichen Lebenskreis ist danach die Bedingung eines andauernden Engagements von sozialprofessioneller Seite.

Nehmen wir die persönliche Sicht, die vorhandene Kundigkeit und das Interesse von Menschen zum Ausgangspunkt, besteht der Einsatz von Diensten zunächst darin, ihre Sorgen zu teilen und sie mit einem vorhandenen oder zu aktivierenden zivilen Engagement zu verbinden. Das kann in dem konkreten, auch räumlich ausgedehnten persönlichen Lebenszusammenhang geschehen: in den Beziehungen des Wohnens und sozialer Kontakte, den Relationen der Arbeit und täglicher Besorgungen. Sozialdienste lassen sich darin »in Lösung bringen «, wenn sie die Gestaltung ihrer Arbeit nach Inhalt und Form den Alltagsverhältnissen und den örtlichen Gegebenheiten anpassen.

Diese Versorgungsgestaltung im Milieu muss nicht von den Dienstleistern initiiert werden; auch deren potenzielle Nutzer können sie in ihrem Lebenskreis und Nachbarschaft in die Wege leiten. Wird jeder lokalen Bereitstellung von Diensten und ihrer Ausformung vor Ort eine Diskussion der Bewohner vorausgesetzt, ob und wie sie stattfinden soll, verlegt man die Verantwortung für ihre Einführung und Nutzung in das Engagement der Bürger und bindet das Bestehen des Versorgungsangebots an ihre Beteiligung (vgl. das Beispiel Barmen im Kasten »Wir sind das Dorf «).

Von sozialprofessioneller Seite ist ein erhebliches »Entgegenkommen« zu erwarten, um Fachdienste in die gemeindliche und milieuspezifisch individuelle Versorgungsgestaltung einzugliedern. Was fachpflegerisch gedacht ist, mutiert dann vielleicht zu einer Unterstützungspraxis von dementiell behinderten alten Menschen und ihren Angehörigen. Und aus dem Angebot einer professionellen Hilfe zur Erziehung wird ein spezieller Service für Mehrkinderfamilien in schlechten Wohnverhältnissen oder für beruflich belastete Alleinerziehende. Eine Kindertagesstätte eignet sich als Anlaufstelle und Knoten eines Elternnetzwerks.

Die Einrichtung oder der Dienst artikuliert die vorgebrachte Problematik und vertritt ihre Behandlung. Ankommen im Milieu heißt so für Institutionen, Austragungsstelle ihm adäquater Lösungen zu werden.

\section{Literatur}

Dörner, Klaus: Leben und sterben, wo ich hingehöre. Dritter Sozialraum und neues Hilfesystem. Paranus Verlag, Neumünster 2007. Dörner, Klaus: Ende der Institutionen? Psychiatrie und Behindertenhilfe im Dritten Sozialraum. In: Evangelische Stiftung Alsterdorf/ Katholische Hochschule für Sozialwesen Berlin (Hg.): Enabling Community. Alsterdorf Verlag, Hamburg 2010. S. 83-91.

Hinte, Wolfgang: Das Fachkonzept "Sozialraumorientierung « als Grundlage für den Umbau der Jugendhilfe. In: Jugendhilfe, 49, 4, 2011. S. 223-230.

Hinte, Wolfgang/Treeß, Helga: Sozialraumorientierung in der Jugendhilfe. Theoretische Grundlagen, Handlungsprinzipien und Praxisbeispiele einer kooperativ-integrativen Pädagogik. 2. Aufl., Juventa, Weinheim 2011. Holtkamp, Lars/Bogumil, Jörg: Bürgerkommune und Local Governance. In: Schwalb, Lilian/ Walk, Heike (Hg.): Local Governance - mehr Transparenz und Bürgernähe? VS, Wiesbaden 2007. S. 231-250.

Krisch, Richard: Sozialräumliche Methodik der Jugendarbeit. Aktivierende Zugänge und praxisleitende Verfahren. Juventa, Weinheim 2009.

Reutlinger, Christian: Sozialpädagogische Räume - sozialräumliche Pädagogik. Chancen und Grenzen der Sozialraumorientierung. In: Deinet, Ulrich/Gilles, Christoph/Knopp, Reinhold (Hg.): Neue Perspektiven in der Sozialraumorientierung. Frank \& Timme, Berlin 2006. S. 23-43

Schiele, Gerhard: Mehrere Partner, ein Ziel: wohnen bleiben im Vertrauten. In: neue caritas, 112, 16, 2011. S. 9-13.

Thomas, Peter Martin/Calmbach, Marc: »Wer ist denn diese sugend ?? Zielgruppen Sozialer Arbeit. In: Forum sozial, 2/2011. S. 11-15

Zukunft Quartier - Lebensräume zum Älterwerden: Themenheft 1. Bertelsmann Stiftung, Gütersloh 2007. 